\title{
DISEÑO DE UN CORREDOR TURÍSTICO ENTRE LOS MUNICIPIOS DE LA CALERA Y SOPÓ, CUNDINAMARCA, COLOMBIA
}

\section{A TOURIST COORIDOR DESIGN BETWEEN TOWNSHIPS OF THE CALERA AND SOPÓ, CUNDINAMARCA, COLOMBIA}

\author{
Jairo Alberto Vásquez Bernal ${ }^{1}$ \\ Adriana Posada Arrubla ${ }^{2}$ \\ María Concepción Salazar Alonso ${ }^{3}$ \\ Doris Emilia Guerrero 4 \\ William Ricardo Zambrano 5 \\ Álvaro Lamprea Delgado ${ }^{6}$ \\ Jorge Sandoval Torres ${ }^{7}$
}

\section{RESUMEN}

El turismo es un subsector de alto crecimiento por los beneficios económicos y sociales que genera, no sólo en los países más adelantados, como los de la Unión Europea y América del Norte, sino en Colombia, por presentar ventajas comparativas derivadas de su posición

${ }^{1}$ Mercadotecnista, Esp Gestión Social y Ambiental Docente Investigador Ingeniería Comercial U.D.C.A. Email: jvasquez@udca.edu.co Dirección para correspondencia: Calle 222 No. 54-25, Bogotá, D.C.

${ }^{2}$ Economista Agrícola M.Sc. Planeación Urbana y Regional, decana Ingeniería Comercial. U.D.C.A. Email: aposada@udca.edu.co

${ }^{3}$ Economista - Bibliotecóloga. Docente Investigador Ingeniería Comercial. U.D.C.A. Email: masalazar@udca.edu.co

${ }^{4}$ Abogada. Filosofa Docente Investigador. Ingenierìa Comercial U.D.C.A. Email: dorisgue@udca.edu.co

${ }^{5}$ Comunicador Social - Periodista M.Sc Comunicación. Docente Investigador Ingeniería Comercial. U.D.C.A. Email: zambrano_william@hotmail.com

${ }^{6}$ Profesional Negocios Internacionales. Docente Investigador. Ingeniería Comercial U.D.C.A. Email:alamprea@udca. edu.co

7 Economista-mercadotecnista M.Sc. Docencia Universitaria. Docente Investigador Ingenierìa Comercial. U.D.C.A. Email: jorhumsan@hotmail.com geográfica y de sus recursos naturales. Un ejemplo específico de ello es el caso de los municipios de Sopó y de La Calera, en el Departamento de Cundinamarca, los cuales por su proximidad con la ciudad de Bogotá, por sus atractivos naturales y por el apoyo de las alcaldías y de la Universidad de Ciencias Aplicadas y Ambientales U.D.C.A, han logrado un proceso para el conocimiento del mercado turístico, dentro del cual se ha podido plantear el diseño de un corredor turístico. Para su diseño, se requirió identificar los elementos, componentes y variables de los servicios turísticos existentes y, posteriormente, establecer un sistema de relaciones utilizando matrices de doble entrada, donde se indicaron los valores agregados de los diferentes tipos de turismo. Al tamizarlos, seleccionarlos y determinarlos con los servicios comunes, se logró definir el corredor, con los atractivos turísticos principales y complementarios. La definición de un corredor turístico, en estas condiciones, ayuda a potencializar el flujo de visitantes con procesos que se van consolidando a través del desarrollo de productos turísticos.

Palabras clave: Corredor turístico, diseño, atractivos, actividades y servicios turísticos.

\section{SUMMARY}

Tourism is a high-growth sector due to its economic and social benefits, not only in the most advanced countries 
like those of the European Union and North America, but also in Colombia, where advantages are added because of its geographical position and natural resources. A specific example is the case of the municipalities of Sopó and La Calera, in the Department of Cundinamarca, which, due to their proximity to Bogotá, their natural attractions, the support of Mayors and the University of Applied Sciences and Environmental U.D.C.A, have developed a knowledge of the tourism market, that made it possible to conform the design of a tourist corridor. For the design of this corridor, the identification of elements and components of tourism services was required and, a system of double entry matrices was established, which indicated the added values of the different tourism opportunities. Afterwards, when confronting them with services, the goal was accomplished by defining the corridor with the most tourist attractions and complementary services. The meaning of a tourism corridor under these conditions helps to enhance the flow of visitors with processes that are consolidating through the development of products for tourism.

Key words: Tourism corridor, design, attractive tourist activities, and services.

\section{INTRODUCCIÓN}

El sector dentro del cual se encuentra el turismo, ha experimentado un desarrollo amplio en las últimas décadas, representando el $65 \%$ del producto global y el $25 \%$ de las transacciones mundiales (Vargas \& Aldana, 2007). Es el sector de más rápido crecimiento en la mayoría de economías del mundo (Walker et al. 2005), por ejemplo, en Estados Unidos representa el $76 \%$ del producto interno bruto y el $79 \%$ del empleo (Lamb et al. 2006). El turismo, se ha convertido en un negocio global, cuyo mercado en expansión cubre prácticamente todos los lugares y destinos. Más de 625 millones de turistas viajaron fuera de su país y gastaron 444.000 millones de euros. Esta actividad representa el $31 \%$ del comercio internacional y más de 100 millones de puestos de trabajo (Kotler et al. 2005).

En Brasil y otros países suramericanos, el turismo es un importante sector de la actividad económica. Su contribución a la creación de riqueza y a mejorar el bienestar de los ciudadanos, se puede sentir de muchas maneras: a través de la generación de empleo, distribución de ingresos, preservación del medio y la restauración del patrimonio histórico y cultural (Oliveira, 2007).
En Colombia, el Ministerio de Comercio, Industria y Turismo (2004) desarrolló el estudio de competitividad del turismo y estableció que la participación del sector al Producto Interno Bruto (PIB) no ha pasado de 3\%, mientras que en países, como España, representa el $18 \%$ y del Caribe, hasta el $30 \%$. De la población económicamente activa del país, el $10 \%$ se concentra en las actividades turísticas y en el contexto de la economía; el turismo aporta al volumen total de exportaciones, ocupando el tercer lugar después del café.

El ecoturismo es importante para el desarrollo económico y ambiental, asílo define Caicheo (2006), como las actividades en las cuales se privilegian la preservación y la apreciación del medio que acoge a los viajantes. Al respecto Jax \& Rozzi (2004) establecen que la conservación es una tarea compleja que involucra tanto valores sociales, teorías ecológicas e interés por el patrimonio natural y cultural. Kent (2003) indica que el ecoturismo funciona como un claro catalizador del cambio, en el sentido de que incorpora nuevos entornos en las economías de mercado. En la zona rural, se evidencian los cambios relacionados con el ecoturismo, como lo establece Martínez (2004), mencionando que la gestión de los recursos naturales constituye la base de muchos impactos biológicos, sociales y políticos. Arias (2005), afirma que son espacios en los cuales el hombre hace encuentro entre lo político, lo social, lo cultural, lo económico, lo productivo y lo natural

Por lo tanto, el turismo y el ecoturismo, se constituyen en un factor de crecimiento económico por los impactos, especialmente en el empleo y los ingresos, por lo cual, los países más desarrollados establecen normas, políticas y proyectos para fortalecerlo. Colombia, cuenta con la Ley 300 de 1996, Ley General de Turismo y sus modificaciones en la Ley 1101 de 2006.

Es importante destacar que en el departamento de Cundinamarca, cuenta con planes turísticos, enmarcados en el Plan de Desarrollo Nacional, pero no han logrado un crecimiento en los últimos años. De la misma manera, el problema se presenta en los municipios de La Calera y Sopó, lo cual se debe a que no han tenido una administración, fomento y desarrollo óptimo de sus recursos naturales, de sus sitios históricos y culturales, lo que justifica el diseño del corredor turístico.

La Gobernación de Cundinamarca (2004) estructuró un Marco de Competitividad y Cooperación Técnica 
para desarrollar acciones que fortalezcan el progreso del turismo regional; de la misma manera, en el plan de desarrollo de Bogotá (2004), refuerzan el programa de turismo, ratificando la necesidad del corredor turístico, especialmente, en los municipios vecinos, y para este caso Sopó y La Calera, por sus condiciones y características.

Dado que un corredor turístico es un espacio biofísico con características geográficas especiales donde se encuentran una serie de atractivos naturales, culturales y de servicios turísticos, interconectados para el beneficio de la sociedad es que se realizó esta investigación para definir el mercado turístico, herramienta base para estructurar el diseño del corredor. Se desarrolló, con el reconocimiento de las potencialidades turísticas de los municipios y caracterización de las empresas, las actividades y los servicios que éstos poseían; al resultado de este estudio, se identificaron las variables, elementos y componentes del mercado turístico de Sopó y La Calera, para el diseño del corredor.

El municipio de Sopó, localizado en el valle del río Teusacá, esta ubicado a $39 \mathrm{~km}$. de Bogotá. Tiene una extensión de $113,5 \mathrm{~km}^{2}$, la mayor parte del territorio es plano; se destacan los cerros de Agua Caliente y Pionono (CAR, 1992). El casco urbano de Sopó, se ubica entre los $4^{\circ} 5450^{`}$ de latitud norte y $73^{\circ} 5706^{`}$ de longitud oeste, el promedio de temperatura es de $14^{\circ} \mathrm{C}$ y la pluviosidad anual media es de 978mm (IGAC, 1996).

La Alcaldía de La Calera (2004), en su plan de desarrollo, indica que el municipio está localizado aproximadamente a $16 \mathrm{~km}$ de Bogotá y se caracteriza por estar ubicado en el valle sobre el río Teusacá. Todo su territorio de sur a norte está surcado por la cordillera oriental, la cual presenta numerosas ramificaciones, dando un aspecto bastante quebrado, alternando valles, colinas y elevaciones, contraste admirado por los turistas.

\section{MATERIALES Y MÉTODOS}

El diseño del corredor, se realizó estructurando el sistema de relaciones, en el cual, se integraron las variables establecidas por cada una de las unidades objeto de estudio: comunidad, turistas y empresas, identificando las comunes, a través de un proceso de selección. Igualmente, con la elaboración de tablas, gráficas y resultados, se elaboró un análisis y una interpretación de la información. Con ella, se determinó los nuevos servicios, combinando las variables en matrices de doble entrada de los diferentes servicios turísticos ofrecidos. Así, se identificaron los atractivos, los componentes, los servicios base, los periféricos y los complementarios comunes y representativos, con los que se diseñó el corredor, consolidándolo con propuestas, a través de la construcción de circuitos turísticos y la aproximación a un modelo.

La base para el diseño del corredor fue la necesidad de llevar a la práctica una propuesta interdisciplinaria entre las concepciones de la economía, la comunicación social, el derecho y la mercadotecnia, con el fin de establecer una forma de unir esfuerzos en un sitio determinado, mediante la prestación de servicios turísticos para una comunidad y empresarios que requieren de su desarrollo, sin dejar de lado, la satisfacción de necesidades de los usuarios.

El estudio de mercados sirvió como herramienta cuantitativa (Hernández, 2003; Churchil, 2003), descriptiva (Tamayo, 2002; Lerma, 2002) y aplicada (Ortiz E García, 2003), para la identificación de las variables indispensables para el corredor, utilizando unidades objeto de estudio de Sopó y La Calera. A partir de ello, el tamaño de muestra, se obtuvo por la distribución muestral de las proporciones (McDaniel E Gates, 2006; Hair et al. 2004) y para los usuarios de los servicios, se aplicó la fórmula:

$$
\mathrm{n}=\frac{\mathrm{P} \times \mathrm{Q} \times \mathrm{Z}^{\underline{2}}}{\mathrm{E}^{2}}
$$

La determinación de la muestra para las empresas públicas y privadas de los municipios y de la comunidad que son finitas, se aplicó la fórmula:

$$
\mathrm{n}=\frac{Z^{2} \times P \times Q \times N}{E^{2}(N-1)+Z^{2} \times P \times Q}
$$

El tamaño resultante de muestra, representativo por poblaciones, fue el siguiente: para Sopó: comunidad 76, empresas 52 y turistas 105. Para la Calera estos números correspondieron, respectivamente, a 88, 73 y 115.

El proceso de selección de las unidades de estudio (Zikmund, 2003; Kinnear E Taylor, 1998; Jany, 2005), se realizó a través de un muestreo aleatorio simple por retención para los turistas, estratificado por tipos a las 
empresas y por conglomerados a la comunidad, en el cual, la población se dividió en grupos hasta llegar a las cuadras o veredas.

La recolección de los datos, se realizó en formatos (Cuadros 1, 2 y 3) estructurados directos relacionados con el conocimiento, las actividades, los atractivos, los servicios turísticos, principalmente, con preguntas abiertas y cerradas, en encuestas específicas en formatos finales para las unidades de estudio, las empresas turistas y la comunidad. El registro de la información, se realizó a través de entrevistas personales y los datos se editaron, tabularon y graficaron, lo que permitió determinar los porcentajes que se obtuvieron de acuerdo a la cantidad de respuestas por pregunta en proporción directa al tamaño de muestra, realizando la descripción, análisis e interpretación de resultados.

Luego, con la combinación, la esquematización y la diferenciación de los servicios, se logró la elaboración de matrices de doble entrada, que establecen nuevos servicios para los visitantes, los empresarios y la comunidad. Los seleccionados se graficaron, integrándolos para identificar solo aquellos que tienen potencial en los municipios, para finalmente, por medio de un proceso lógico, abstraer los elementos del servicio para hacer una propuesta de aproximación a un modelo de corredor turístico.

Cuadro 1. Formato final aplicado a empresas públicas y privadas.

Empresa:

Fecha:
Nombre:

Dirección:
Cargo:

Teléfono:
Vereda:

1. ¿Ofrece usted servicios a turistas? $1.1 \mathrm{Si}$

1.2 No

2. ¿Cuáles son los servicios turísticos que ofrece?

3. ¿Qué días ofrece los servicios turísticos?
3.1 Todos los días
3.2 Fines de semana
3.3 Otros
Cuáles

4. ¿Cuántas personas trabajan en su establecimiento?
4. 1 1-4
4.2 5-8
4.3 9-12
4.4 13-16
4.5 otro
Cuántos

5. ¿Cuál es la cantidad promedio de personas que puede atender en un día?
$5.11-25$
$5.226-50$
$5.351-75$
$5.475-100$
5.5 Otra

6. ¿Cuál es el costo promedio de los servicios que ofrece?

7. Los clientes, consumidores o usuarios de su establecimiento son:
7.1 Turistas

7.2 Residentes

7.3 Otros

Cuáles

8. ¿Cuáles son los factores de éxito en el desarrollo de su negocio?

9. ¿Apoya o contribuye a eventos?

9.1 Sí 9.2 No

10. ¿¿Usted contribuye en algún evento como apoyo al turismo en el municipio?

11. ¿Promociona los servicios turísticos a sus visitantes?

11.1 Sí $11.2 \mathrm{No}$

13. ¿Cuándo realiza esta promoción menciona en qué eventos o recursos?

14. ¿Cuáles son los recursos ecológicos, arqueológicos, culturales o históricos que se encuentran en los alrededores de las áreas donde presta sus servicios?

15. ¿Participaría en un proyecto para la conformación de un corredor turístico entre los municipios de La Calera y Sopo? 15.1 Sí 15.2 No

16. ¿Participaría en procesos de capacitación y asesoría empresarial?

16.1 Sí 16.2 No 
Cuadro 2. Encuesta final aplicada a los turistas.

Nombre:

Fecha:

Teléfono:

Ciudad:

Dirección:

1. ¿Participa usted en las actividades turísticas? $1.1 \mathrm{Si}$

1.2 No

2 ¿En qué tipo de turismo prefiere participar?

3 ¿Con que personas comparte esta actividad?
3.1 Amigos
3.2 Familia
3.4. Otros
Cuáles

4. ¿Qué otros municipios visita usted?

5. ¿Con qué frecuencia visita usted el municipio?
5.1 Una vez a la semana
5.2 cada 15 días
5.3 una vez al mes
Otra
Cuál?

6. ¿Qué atractivos turísticos visita usted?
6.1 Religiosos
6.2 Ecológicos
6.3 Monumentos
6.4 Ferias
6.5 Otro
Cuál
7.4 Tienda artesanal
7.1 Guianza
7.2 Gastronómicos
7.3 Transporte
7.5 Deportes extremos 7.6 Otros

8. Cuánto dinero invierte cuando realiza turismo:

9. Si existiera un corredor turístico entre los municipios de Sopó y La Calera, usted lo visitaría?

$9.1 \mathrm{Si}$

9.2 No

Cuadro 3. Encuesta final aplicada a la comunidad.

Vereda :

Dirección:
Nombre :

Fecha:
Teléfono:

1. ¿Conoce las actividades turísticas que se realizan en el municipio?

$1.1 \mathrm{Si}$

1.2 No

2. ¿Cuáles son las actividades turísticas que realizan?
2.1 Caminatas
2.2 Cabalgatas
2.3 Ciclopaseos

2.4 Visita religiosa

2 5. Otros

Cuáles

3. ¿En cuales de las actividades mencionadas participa?

3.1 Caminatas 3.2 Cabalgatas

3.3 Ciclopaseos

3.4 Visita religiosas

3.5. Otros

4. ¿Sabe usted que es Ecoturismo? 4.1 Si

$4.2 \mathrm{No}$

5. ¿Conoce actividades de ecoturismo realizadas ? $5.1 \mathrm{Si}$ 5.2 No

6. ¿Cuáles son las actividades de ecoturismo que se realizan en el municipio?

7. ¿Cuáles son los atractivos ecoturísticos de Sopó?

8. ¿Da a conocer los atractivos ecoturísticos?

8.1 Sí

8.2 No

9. ¿Cuáles son vías de acceso al municipio:
9.1 Briceño
9.2 La Calera
9.3 Tocancipa
9.4 Otra

10. Usted cree que el día de mayor afluencia de turistas corresponde a:

10.1 Sábado

10.2 Domingo

10.3 Lunes Festivo

10.4 Otro

11. ¿Los turistas deterioran los lugares naturales del municipio?

11.1 Si _ Cuáles son los daños

$11.2 \mathrm{No}$

12. ¿Considera que la afluencia de turistas es importante para el municipio? $12.1 \mathrm{Si}$

13. ¿Cuál cree usted son los beneficios que trae el turismo?

13.1 Desarrollo económico __ 13.2 Desarrollo social

13.3 Publicidad 13.4 Otro

14. ¿Participaría usted en un proyecto para la conformación de un corredor turístico? $14.1 \mathrm{Si}$

14.2 No 


\section{RESULTADOS Y DISCUSIÓN}

Los resultados generados en la investigación de mercados permitieron identificar los elementos para el diseño del corredor, los cuales comprenden la estructura, el boceto o el patrón donde se indican las variables y/o competentes de los servicios turísticos ofrecidos en los municipios de Sopó y La Calera. Las variables son los tipos, las actividades y los eventos turísticos, que permiten configurar los sistemas de relaciones, donde se determinan y describen las interrelaciones de los servicios de las unidades objeto de estudio.
Después de analizar las posibilidades empresariales de desarrollo del turismo, se estructuró el diseño del corredor. Los resultados del estudio de mercados se integraron y de ello se generó un esquema inicial, como se observa en la gráfica 1 .

La información obtenida de los usuarios se integró, con el fin de estructurar el sistema de relaciones, con lo cual se obtuvo que el $85 \%$ visitan los municipios, el $35,1 \%$ practican turismo ecológico, el $15,6 \%$, turismo de aventura y el $13,4 \%$, asisten a los lugares religiosos. Los datos muestran un potencial alto por parte de visitantes

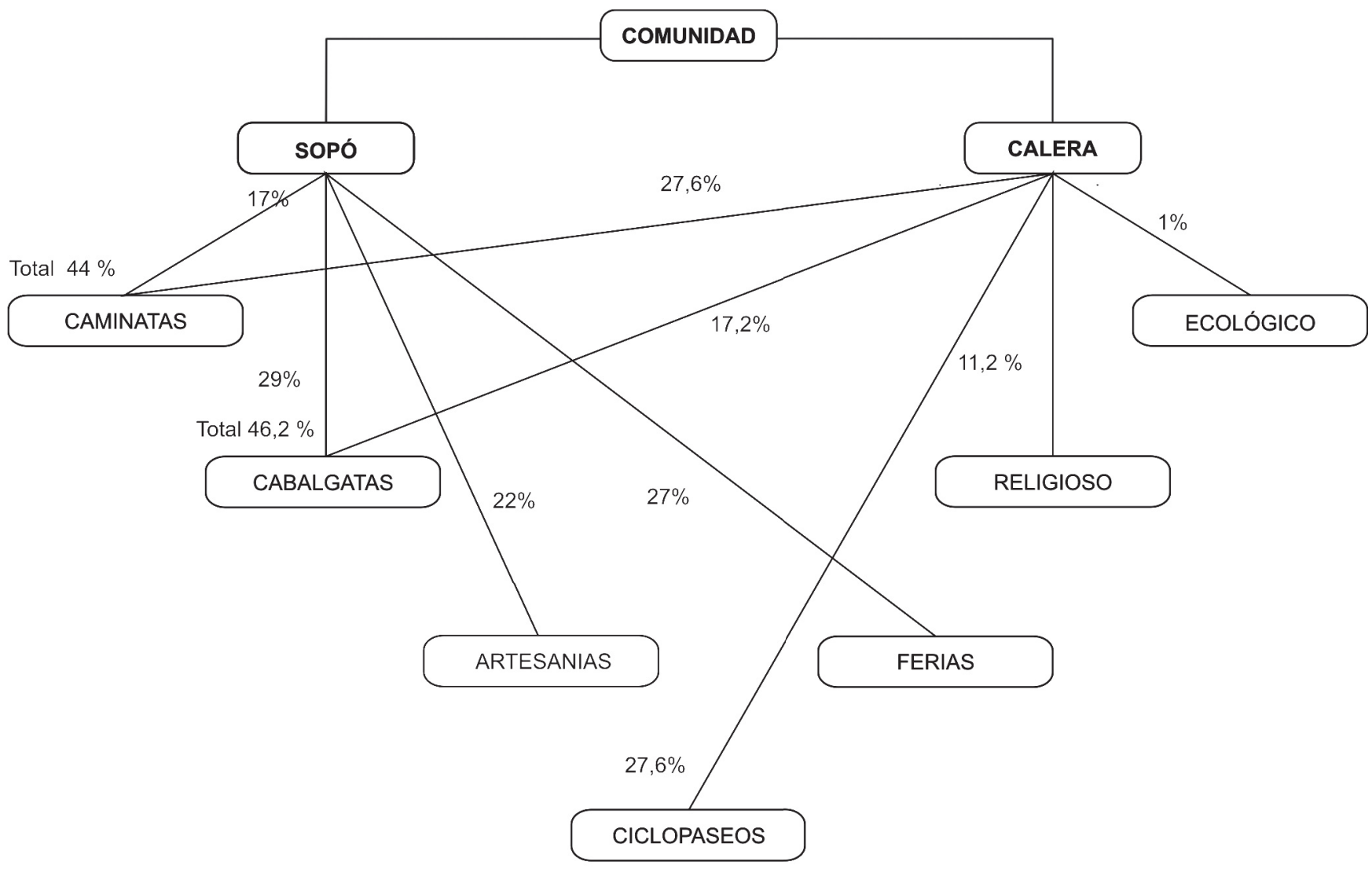

Gráfica 1. Integración del sistema de relaciones para la comunidad

interesados en conocer y participar en diferentes tipos de turismo relacionado con la naturaleza.

Al integrar los resultados del estudio a las empresas públicas y privadas, se obtiene que el $95,2 \%$ de empresarios de los municipios ofrecen servicios a los turistas; el 23,2\% de gastronomía y el $8 \%$ de cafetería; el $84 \%$ de los empresarios prestan servicio todos los días. Las actividades que más promocionan los empresarios son las ferias y fiestas, con un 19,2\%, y los recursos naturales como el embalse de San Rafael, con 16,50\%, lo que indica que es factible el diseño del corredor por la variedad de servicios que se ofrecen, la motivación de los empresarios en promocionar el turismo y los recursos disponibles.

Con lo anterior, se construyó el sistema de relaciones con la integración de los resultados de la comunidad, 
los turistas y las empresas, permitiendo describir la estructura del proceso turístico, con base en las interrelaciones de los servicios que más porcentaje representan de las unidades objeto de estudio.

El sistema de relaciones aplicado a los resultados del estudio en los municipios de Sopó y La Calera destaca, por parte de los empresarios, los servicios gastronómicos y ecológicos; de los visitantes, el turismo ecológico, gastronómico y religioso y respecto a la comunidad, alimentación, caminatas y cabalgatas,

Las interrelaciones, resultado de las respuestas obtenidas por unidades objeto de estudio en los municipios, orientan el diseño del corredor hacia los servicios ecológicos y gastronómicos. Se le adicionaron valores agregados y estructuración de servicios, con elaboración de matrices de doble entrada para establecer nuevas alternativas, como combinaciones, donde $\mathrm{R}$ es la ruta, el número es la cantidad de alternativas y las letras la secuencia, entonces quedan representadas así:

R1A: primera relación, en el municipio inicia el servicio y realiza caminata

R1B: segunda relación, realizada la caminata, llega al municipio

R1C: tercera relación, en el municipio, se dedica a la cabalgata

R1D: cuarta relación terminada cabalgata, se da fin al servicio.

Secuencialmente y de esta manera, se realizaron las diferentes posibilidades de combinaciones de servicios con los atractivos y tipos de actividades turísticas.

La matriz con los datos de la comunidad determinó la estructura de combinaciones de servicios, establecidos por los tipos, las actividades o atractivos turísticos, las cuales presentan 15 alternativas diferentes; los valores agregados que se pueden ofrecer son los siguientes: caminatas religiosas y artesanales; actividades religiosas, ecológicas y culturales, entre otras, ya que cada variable al relacionarse con otra posibilita nuevas actividades. La combinación de servicios turísticos en la matriz relacionados con las empresas permite considerar 17 arreglos distintos, que se consideran valores agregados, como deportes ecológicos y culturales y actividades religiosas, ecológicas y gastronómicas, entre otras.
Los servicios turísticos comunes para los turistas que visitan ambos municipios en la matriz, se establecieron en seis combinaciones factibles, como turismo religiosoecológico-gastronómico; religiosos-cultural y ecológico. La matriz que relaciona los municipios y las unidades objeto de estudio dan como resultado 27 combinaciones diferentes, con servicios, como deportivos, religiosos y gastronómicos; también se pueden encontrar otras alternativas, como actividades ecológicas, culturales y religiosas; caminatas culturales y gastronómicas; cabalgatas, culturales y religiosas y otras.

Para la propuesta del diseño del corredor turístico, se tamizó por el resultado de la integración de variables y las alternativas factibles de las matrices, lo que permite consolidar el sistema de relaciones, como se observa en la gráfica 2, en la cual, se destaca los servicios turísticos comunes entre las unidades objeto de estudio comunidad y empresas y entre los usuarios son los ecológicos y los actividades religiosas, que se deben considerar en el diseño del corredor.

La estructuración del corredor, se realiza incluyendo los atractivos naturales, las actividades y servicios que se interrelacionaron, como: Pionono, $4 \%$, San Rafael, 3,5\%, Chingaza, 2,3\% y parque CAR Sopó, 3,5\%, entre los recursos ecológicos; Iglesia Divino Salvador, 2,8 \%, santuario del señor de la piedra, 2,8\%, arcángeles de Sopó, $1,2 \%$ y capilla colonial, $2,9 \%$ entre los religiosos y los servicios gastronómicos, lo cual corresponde a la parte final del diseño, con aquellas combinaciones de relaciones que dan cuenta de la lógica interna del corredor.

Una alternativa que permita dar valor agregado a los servicios turísticos del corredor es la combinación de los tipos de turismo comunes, esto refleja el resultado del estudio donde se establecieron diversos gustos por servicios, en otras palabras, el corredor no puede ser monotemático y debe ofrecer diversidad de posibilidades de servicios. Aunque existen muchas alternativas, se escogió el de La Calera por su facilidades, atractivos y servicios, con los cuales establece el corredor, que se diseño de la siguiente manera: inicia cuando los turistas salen de Bogotá, por ser la capital del país y donde se encuentra la mayoría de visitantes que se dirigen a los municipios, realizando actividades y visitas, como son en su orden geográfico: Embalse de San Rafael, Iglesia, Capilla Colonial y restaurantes en La Calera, Parque 


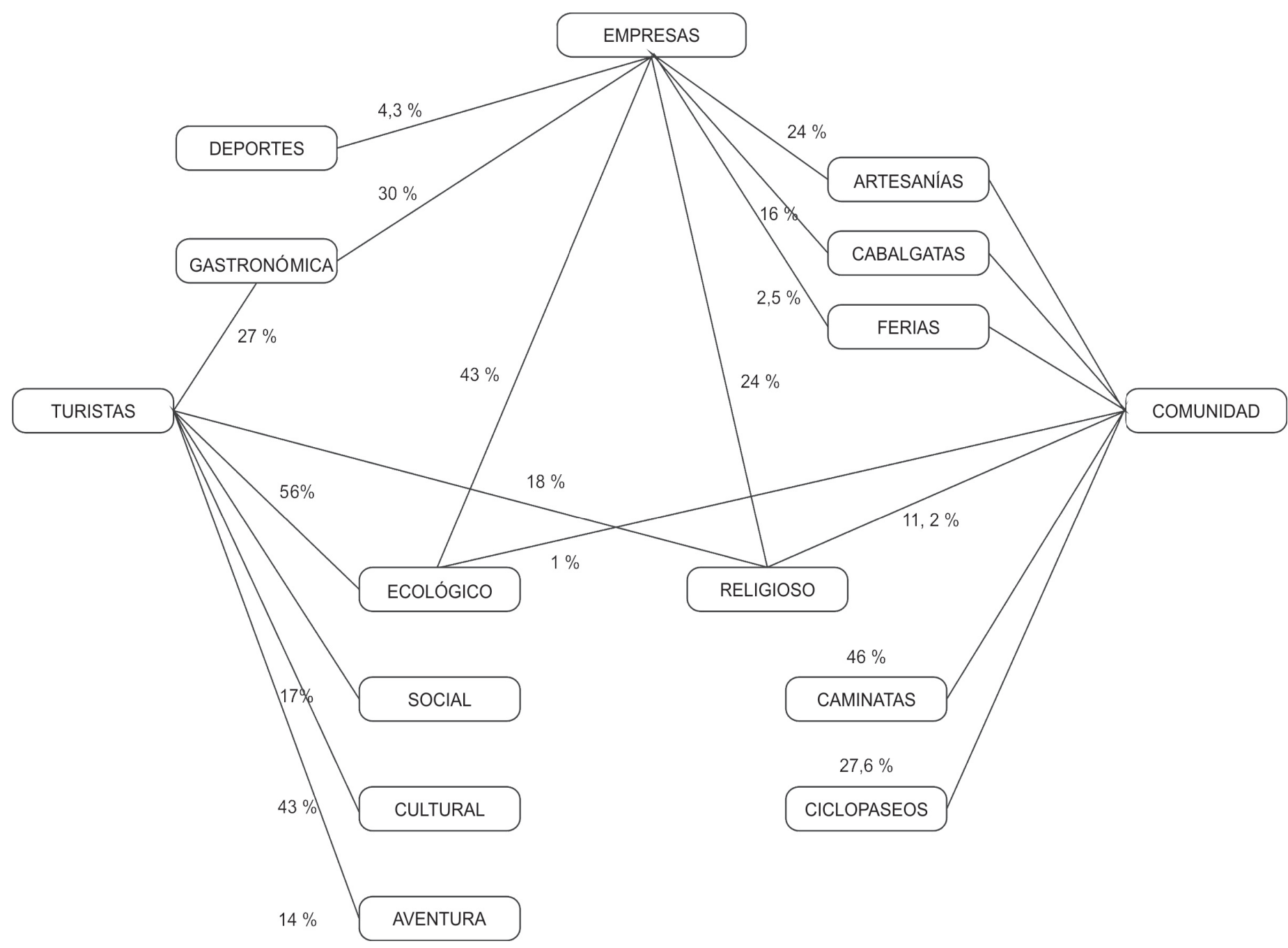

Gráfica 2. Consolidación de la integración del sistema de relaciones y matrices.

Natural Chingaza, atractivo turístico Pionono, iglesia del Divino salvador, capillas de los Arcángeles y Colonial, Santuario del señor de la Piedra en Sopó, restaurantes Briceño, Parque de la CAR y regreso a Bogotá.

Igualmente, en el mapa, se encuentran los circuitos que componen el diseño del corredor de los municipios de Sopó y La Calera por el sistema de relaciones, donde se integraron los servicios turísticos ecológicos, gastronómicos y religiosos; el turista define la posibilidad que en el corredor se propongan unos circuitos adicionales, pero precisándolos con las necesidades del turistas, la frecuencia de visita y los lugares; a manera de ejemplo:

Circuito 1. Bogotá - Calera - Templo - Restaurante - San Rafael - Bogotá.
Circuito 2. Bogotá - Calera - Capilla - Chingaza Restaurante - Bogotá.

Circuito 3. Bogotá - Sopó - Iglesia - Restaurante - Pionono - Bogotá.

Circuito 4. Bogotá - Sopó - Santuario - Pionono Restaurante - Bogotá.

A partir del diseño del corredor con las actividades, tipos y atractivos turísticos es posible abstraer el proceso y plasmarlo en una aproximación a un modelo de corredor (gráfica 3), destacando que el mercado turístico se conforma de variables, elementos y componentes, en donde interactúan clientes y proveedores en un sistema de relaciones y de combinaciones de los atractivos, servicios y actividades, se interrelacionan permitiendo la estructuración del corredor. 


\section{CONCLUSIONES}

De acuerdo con la contextualizacion, el turismo es una de las actividades económicas que más se ha venido desarrollando e impulsado, convirtiéndose en sinónimo de crecimiento y fuente de ingresos para los países en vías de desarrollo, como Colombia. Por tal razón, las regiones y los municipios como Sopó y La Calera, no se escapan a esta posibilidad de desarrollo.

Los atractivos y los servicios turísticos existentes en los municipios de Sopó y La Calera y su cercanía con Bogotá, los convierte en una alternativa para el desarrollo económico y social, siempre y cuando los elementos, variables y componentes del mercado turístico, como son los atractivos, actividades y servicios relacionados con el subsector, se integren a través de un corredor.

El proceso de relaciones, combinaciones, tamizado e interacciones de los atractivos, actividades y servicios turísticos permitió el diseño del corredor turístico, dando como alternativa una propuesta relacionada con los servicios comunes que se ofrecen en los municipios y que demandan los turistas y son el ecológico, religioso y gastronómico.

Se determinó como componente principal de los servicios turísticos resultado del sistema de relaciones a los recursos naturales, ya que son los atractivos ecológicos los más visitados y reconocidos en los

\section{MERCADO TURÍSTICO}

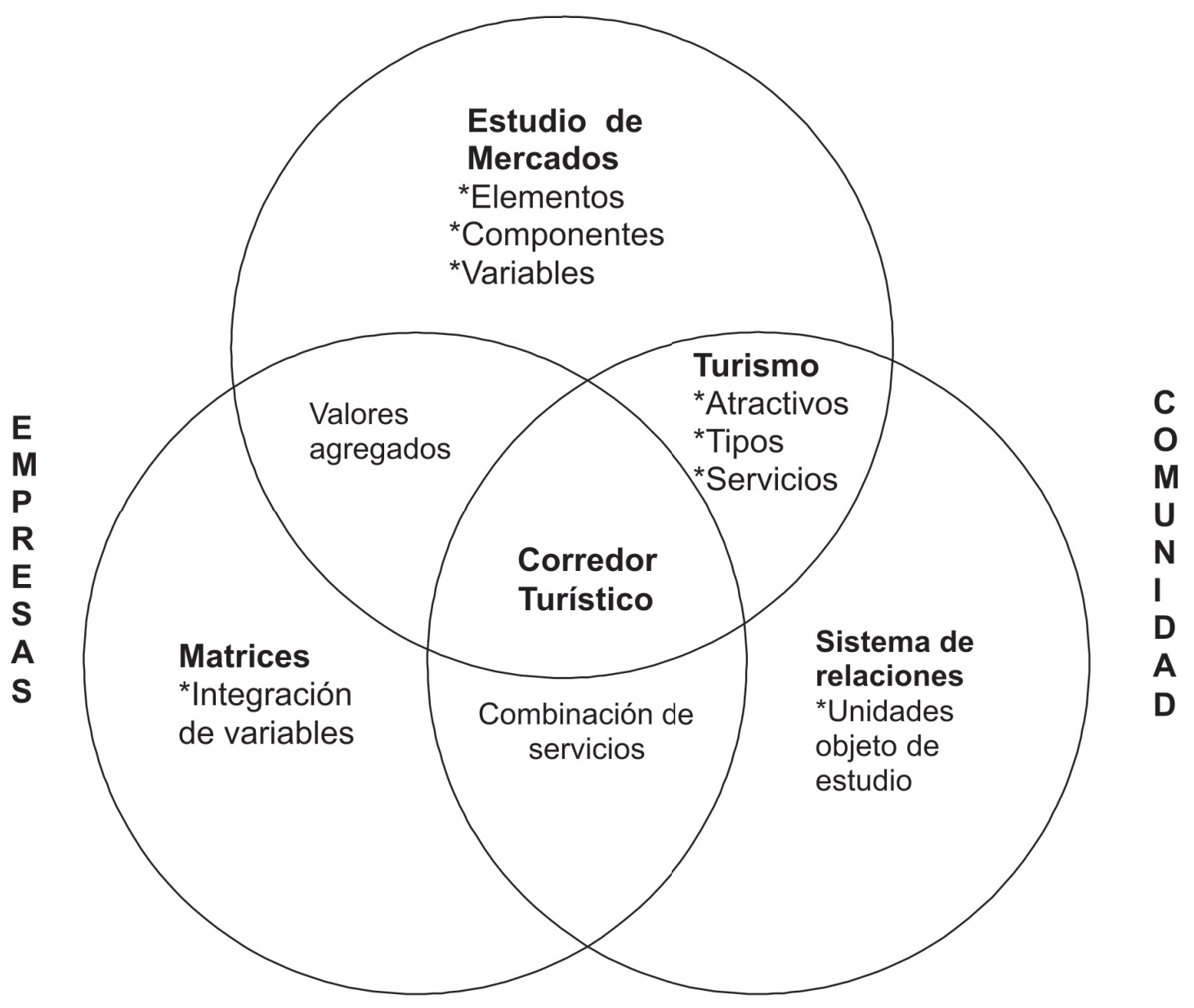

TURISTAS

Gráfica 3. Aproximación a un modelo de corredor turístico. 
municipios de Sopó y La Calera. A nivel religioso, las parroquias de los municipios y sus capillas, sitios base para la estructuración del corredor y de los circuitos.

La implementación, como aproximación al modelo del corredor turístico, es resultado de la abstracción y síntesis del mercado turístico y de las interrelaciones con la comunidad, los turistas y las empresas con la identificación, componentes básicos: estudio de mercados, sistema de relaciones, matrices, combinaciones y valores agregados que integran la propuesta.

\section{BIBLIOGRAFÍA}

ALCALDÍA DE LA CALERA. 2004. Plan de desarrollo Municipio de La Calera 2004-2007. La Calera: La Alcaldía. 145p.

ALCALDÍA DE BOGOTÁ. 2004. Plan de desarrollo económico, social y de obras públicas 2004-2008. Bogotá sin indiferencia un compromiso social contra la pobreza y la exclusión. Bogotá. D.C.: La Alcaldía. 154p.

ARIAS M., I.A. 2005 Apuntes para una discusión sobre desarrollo rural en Venezuela. Espacios. 26(2):1-1.

CAICHEO, A.; GONZÁLEZ, M. 2006. Un esfuerzo de conservación en el fin del mundo. Reserva de biosfera cabo de hornos. Una propuesta de conservación y turismo para el desarrollo sustentable en el extremo austral de América. Magallania. 34(2):131-135.

CORPORACIÓN AUTÓNOMA REGIONAL DE CUNDINAMARCA. CAR. 1992. Atlas Regional. Bogotá: El Instituto. 287p.

CHURCHIL, G. 2003. Investigación de Mercados. 4 ed. International Thomson Editores. México. 830p.

GOBERNACIÓN DE CUNDINAMARCA. 2004. Plan de Desarrollo Departamental Cundinamarca, es tiempo de crecer 2004/2008. Bogotá: La Gobernación. 173p.

HAIR, J.; BUSH, R.; ORTINAU, D. 2004. Investigación de Mercados $2^{\mathrm{a}}$ ed. Mc Graw Hill Interamericana Editores. México. 715p.
HERNÁNDEZ, R 2003. Metodología de la Investigación. $3^{a}$ ed. Mc Graw Hill. México. 705p.

INSTITUTO GEOGRÁFICO AGUSTÍN CODAZZI IGAC. 1996. Diccionario Geográfico de Colombia. Bogotá. Colombia. 897p.

JANY, J. 2005. Investigación Integral de Mercados: Un enfoque para el siglo 21. $3^{\mathrm{a}}$ ed. Editorial Mc Graw Hill. Santafé de Bogotá. D.C. 481p.

JAX, K.; ROZZI, R. 2004. Teoría ecológica y valores en la definición de objetivos de conservación: ejemplos de regiones templadas de Alemania, Estados Unidos de América y Chile. Rev.Chil. Hist. Nat. 77(2):349-366.

KINNEAR, T; TAYLOR, J. 1998. Investigación de Mercados. $5^{\mathrm{a}}$ ed. Santafé de Bogotá. D.C.: Editorial Mc Graw Hill. 874p.

KENT, M. 2003. Ecotourism, environmental preservation and conflicts over natural resources. Horizont. Antropol. 9(20):185-203.

KOTLER, P.; BOWEN, J.; MAKENS, J. 2005. Marketing para el turismo. $3^{a}$ ed. Pearson Prentice Hall. Madrid (España). 565p.

LAMB, C.; HAIR, J.; MCDANIEL. C. 2006. Fundamentos de Marketing. $4^{\mathrm{a}}$ ed. México: Thomson. 591p.

LERMA., H. D. 2002. Metodología de la Investigación. Ecoe ed. Bogotá. 222p.

MARTÍNEZ, Z. 2004. Cogestión de recursos naturales en la Región Atlántica de Colombia. Innovar. 14(23):158-167.

MCDANIEL, C.; GATES, R. 2006. Investigación de Mercados Contemporánea. $6^{a}$ ed. Internacional Thomson Editores. Bogotá. 760p.

MINISTERIO DE COMERCIO, INDUSTRIA Y TURISMO. 2004. Estudio de competitividad del turismo. Bogotá. Colombia. 159p.

OLIVEIRA, E. 2007. Impactos socioambientaís e economicos do turismo e as suas repercussöes no desemvolvimento local: o caso do municipio de 
Itacaré - Bahia. Intercoröes (Campo Grande). 8(2):349-356.

ORTIZ, F.; GARCÍA, M. 2003. Metodología de la investigación: el proceso y sus técnicas. $3^{a}$ ed. México. Limusa Noriega Ed. 279p.

TAMAYO, M. 2002. El proceso de la Investigación Científica. $4^{a}$ ed. Limusa Noriega Ed. México. 231p

VARGAS, M.; ALDANA, L. 2007. Calidad y Servicio; conceptos y herramientas. Ecoe Ed. Bogotá, D. C. 152p.
WALKER, O.; BOYD, H.; MULLINS J.; LARECHE, J. 2005. Marketing Estratégico: un enfoque para la toma de decisiones. $4^{a}$ ed. Mc Graw Hill. 431p.

ZIKMUND, W.G. 2003 Investigación de Mercados. 6a ed. Prentince Hall Hispanoamericana. México. 739 p.

Recibido: Enero 28 de 2008

Aceptado: Mayo 9 de 2008 